\title{
Quiste de duplicación gástrica: inusual presentación en adultos, tratado con éxito por laparoscopía
}

\author{
Gastric duplication cyst: unusual presentation in adults, successfully treated by \\ laparoscopy
}

\author{
Martín A. Ramírez-Morín ${ }^{*}$, Rodrigo Gómez-Álvarez, Ignacio Fonseca-Sada ${ }^{1}$ \\ Aracely A. Guerrero-Arroyo², Francisco Vásquez-Fernández ${ }^{1}$ y Gerardo E. Muñoz-Maldonado ${ }^{1}$ \\ ${ }^{1}$ Servicio de Cirugía General; ${ }^{2}$ Facultad de Medicina. Hospital Universitario José Eleuterio González, Universidad Autónoma de Nuevo León, \\ Monterrey, Nuevo León, México
}

\section{Resumen}

Las duplicaciones gastrointestinales son malformaciones congénitas raras que pueden presentarse en diferentes partes del tracto gastrointestinal, de tamaño y sintomatología variable. Las duplicaciones gástricas representan el 3-5\% de estas malformaciones. La presentación más frecuente es la quística, en la mayoría de los casos en la curvatura mayor. El diagnóstico es raro en la edad adulta y el tratamiento de elección es quirúrgico, el cual puede llevarse a cabo de forma convencional o por mínima invasión. Se presenta el caso de una mujer adulta con diagnóstico de quiste de duplicación gástrica en fondo gástrico adyacente a la unión gastroesofágica a quien se realizó resección total por laparoscopia.

Palabras clave: Anomalía congénita. Laparoscopia. Quiste de duplicación gástrica.

\begin{abstract}
Gastrointestinal duplications are rare congenital malformations, they can appear in different parts of the gastrointestinal tract, of variable size and symptomatology. Gastric duplications represent 3-5\% of these malformations, the most frequent presentation is statistical, located mostly in the greater curvature, the diagnosis is rare in adulthood, the treatment of choice is surgical, which can be carried out in a conventional or minimally invasive manner. We present the case of an adult woman diagnosed with a gastric duplication cyst in the gastric fundus adjacent to the gastroesophageal junction, who underwent total resection by laparoscopy.
\end{abstract}

Key words: Congenital anomaly. Laparoscopy. Gastric duplication cyst.

\section{Introducción}

El quiste de duplicación gástrica es una malformación congénita de rara presentación en adultos ${ }^{1,2}$. En el
$50 \%$ de los pacientes, los quistes son diagnosticados dentro de los primeros años de vida con síntomas como vómitos, dolor abdominal y pérdida de peso; más del $70 \%$ de los casos reportados se describen en pacientes menores de 12 años ${ }^{1,2}$. En adultos, la

\section{Correspondencia:}

*Martín A. Ramírez-Morín

Avda. Dr. José Eleuterio González, s/n

Mitras Centro

Fecha de recepción: 18-03-2020

C.P. 64460 , Monterrey, N.L., México

E-mail: mramirezuanl@gmail.com
Fecha de aceptación: 29-07-2020

DOI: 10.24875/CIRU.20000203
Cir Cir. 2020;88(S2):35-37

Contents available at PubMed www.cirugiaycirujanos.com 0009-7411/C 2020 Academia Mexicana de Cirugía. Publicado por Permanyer. Este es un artículo open access bajo la licencia CC BY-NC-ND (http://creativecommons.org/licenses/by-nc-nd/4.0/). 
mayoría de los casos se descubren incidentalmente por estudios de imagen o por endoscopia gástrica ${ }^{1,3}$.

El diagnóstico preciso de estos quistes antes de la resección es difícil. Los diagnósticos diferenciales son variados, incluyendo tumores del estroma gastrointestinal, tumores neuroendocrinos, heterotopía pancreática, pseudoquistes pancreáticos y tumores neurogénicos ${ }^{1,4}$. Los quistes de duplicación gástrica son sumamente complicados de diagnosticar de forma preoperatoria. Presentamos el caso de una paciente adulta con quiste de duplicación gástrica resecado por laparoscopia de forma exitosa.

\section{Caso clínico}

Mujer de 41 años sin antecedentes patológicos de importancia, que inició su padecimiento 3 días antes de su ingreso al departamento de urgencias por cuadro de dolor abdominal en epigastrio, con irradiación a mesogastrio, de intensidad 8/10, acompañado de vómito en dos episodios; negó fiebre, coluria y acolia. En la exploración física se encontró consciente, cooperadora y hemodinámicamente estable. Se identifica dolor en epigastrio a la palpación profunda, así como signo de Murphy de características dudosas, sin datos de irritación peritoneal en el resto de la exploración abdominal. Se realizó ultrasonido de abdomen que evidenció una vesícula biliar con pared de $0.29 \mathrm{~cm}$ asociada a lodo biliar y un colédoco de $0.3 \mathrm{~cm}$. La paciente continuó con dolor abdominal en el epigastrio y se realizó tomografía de abdomen contrastada (Fig. 1 a), que mostró engrosamiento de la pared gástrica y quiste (Fig. 1 a) de inclusión peritoneal en fondo gástrico adyacente a la unión gastroesofágica. En dicho estudio se observó una inadecuada repleción con contenido gástrico en el interior, gas en la pared que diseca hacia otra cavidad con aire en su interior y con mismo reforzamiento que la pared gástrica normal y dependiente del estómago; no se identificó infiltración a estructuras vecinas. Posteriormente se realizó endoscopia superior sin evidencia de comunicación a lesión quística, se descartó enfermedad ulcero-péptica, y se programó para colecistectomía y resección de quiste de duplicación gástrica laparoscópica (Fig. 1). El procedimiento se llevó a cabo en posición francesa sin eventualidades y se envió la lesión quística para su estudio anatomopatológico (Fig. 2).

La paciente cursó el posoperatorio sin complicaciones, encontrándose afebril, tolerando la vía oral y sin dolor abdominal, por lo que fue dada de alta en el cuarto día posquirúrgico. Continuó manejo de forma

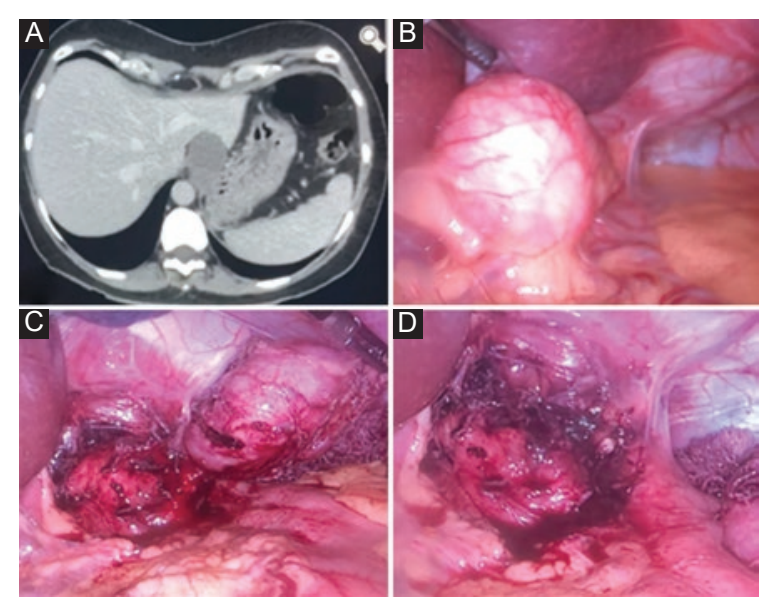

Figura 1. A: tomografía computarizada en la que se observa un quiste de duplicación gástrica. B: quiste de duplicación gástrica en el fondo gástrico. C: resección en proceso. D: lecho quirúrgico posterior a la resección.

ambulatoria, se valoró en su séptimo día posquirúrgico y fue dada de alta definitiva.

El reporte de histopatología fue de colecistitis crónica y quiste de duplicación gástrica de $4.5 \times 3.2 \mathrm{~cm}$. A panorámico $(5 x)$ se observa la pared del quiste, compuesta por músculo liso, y a un mayor acercamiento (40x) se observa una mucosa de aspecto columnar con núcleos alargados, con cromatina fina, moco apical y cilios observados en el epitelio intestinal embrionario.

\section{Discusión}

Las duplicaciones gastrointestinales son malformaciones congénitas raras que pueden desarrollarse en cualquier parte del tracto gastrointestinal, desde el esófago hasta el recto ${ }^{5}$, de tamaño y sintomatología variable. Las duplicaciones gástricas representan el 3-7\% $\%^{1-6}$ de estas malformaciones y la presentación más frecuente es la quística. El diagnóstico es raro en la edad adulta. La mayoría de los casos ocurren en mujeres (8:1)7.

El primer informe en el que se utilizó el término «duplicación intestinal» fue dado por Fitz en 1844, quien sugirió que las duplicaciones del tracto digestivo surgen de la persistencia del conducto onfalomesentéri$\mathrm{Co}^{6}$. El estudio preoperatorio puede incluir ultrasonido abdominal, tomografía computarizada, resonancia magnética y más recientemente ultrasonido endoscópico $0^{8,9}$. La localización más común es la distal en la curvatura mayor; pueden comunicarse con la luz gástrica, pero en la mayoría no se evidencia comunicación hacia la lesión quística, como en nuestra paciente ${ }^{7,10}$. 


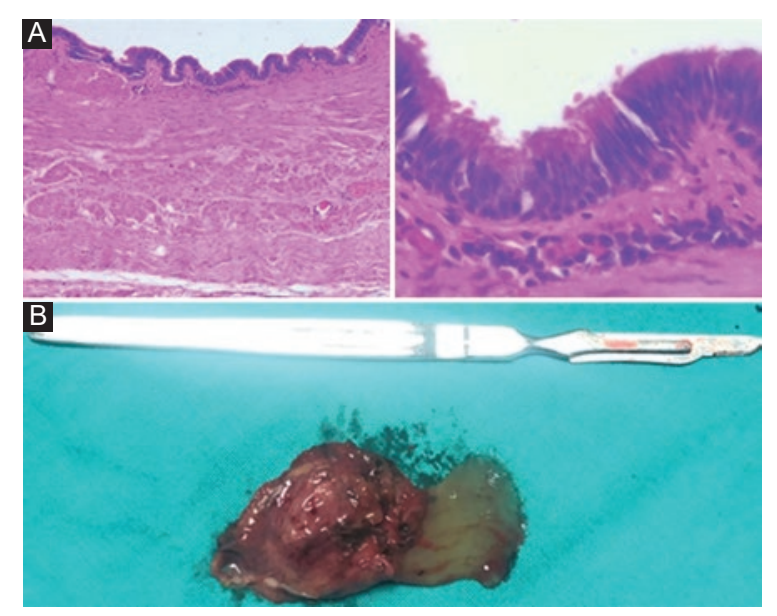

Figura 2. A: corte histopatológico en el que se observa la mucosa tapizada por epitelio monoestratificado cilíndrico alternado con células caliciformes. B: quiste de duplicación gástrica resecado, de contenido mucoide, de $4 \times 4 \mathrm{~cm}$.

Se han reportado numerosas modalidades de tratamiento, que incluyen enucleación, formación de cistogastrostomía e incluso la extracción endoscópica. La piedra angular del tratamiento es la resección quirúrgica del quiste ${ }^{3,7}$. Se recomienda la escisión completa no solo para el alivio sintomático, sino también debido al riesgo de degeneración maligna, que rara vez se informa, pero al menos ha habido $14 \mathrm{ca}$ sos de adenocarcinoma diagnosticados tras la resección de un quiste de duplicación gástrica ${ }^{11}$. Si existe sospecha de transformación maligna, la resección quirúrgica es el tratamiento de elección siempre. Los criterios histológicos para el diagnóstico de un quiste de duplicación gástrica son: a) que la pared del quiste se encuentre contigua a la pared del estómago, b) que el quiste esté rodeado de músculo liso, y c) que la pared del quiste esté cubierta por epitelio gástrico o por cualquier otro tipo de mucosa intestinal ${ }^{12}$.

\section{Conclusión}

Las duplicaciones gástricas, en especial en población adulta, son extremadamente raras; sin embargo, siempre deben formar parte del diagnóstico diferencial de las masas submucosas del tracto gastrointestinal, que incluye lipomas y tumores del estroma gastrointestinal.

\section{Agradecimientos}

Los autores muestran su agradecimiento al servicio de cirugía general del Hospital Universitario José Eleuterio González, a su plantilla de profesores, a los residentes, al personal de enfermería y los médicos internos de pregrado, pues sin el trabajo en equipo nada sería posible.

\section{Conflictos de intereses}

Los autores declaran no tener conflicto de intereses.

\section{Responsabilidades éticas}

Protección de personas y animales. Los autores declaran que para esta investigación no se han realizado experimentos en seres humanos ni en animales.

Confidencialidad de los datos. Los autores declaran que en este artículo no aparecen datos de pacientes.

Derecho a la privacidad y consentimiento informado. Los autores declaran que en este artículo no aparecen datos de pacientes.

\section{Bibliografía}

1. Izumi $H$, Yoshii $H$, Abe R, Mukai M, Nomura E, Ito $H$, et al. Successful laparoscopic resection for gastric duplication cyst: a case report. J Med Case Reports. 2019;13:240.

2. Wieczorek RL, Seidman I, Ranson JH, Ruoff M. Congenital duplication of the stomach: case report and review of the English literature. Am J Gastroenterol. 1984;79:597-602.

3. Perek A, Perek S, Kapan M, Goksoy E. Gastric duplication cyst. Dig Surg. 2000;17:634-6.

4. Kim SM, Ha MH, Seo JE, Kim JE, Min BH, Choi MG, et al. Gastric duplication cysts in adults: a report of three cases. J Gastric Cancer. 2015;15:58-63.

5. Abdalkader M, Al Hassan S, Taha A, Nica I. Complicated gastric duplication cyst in an adult patient: uncommon presentation of an uncommon disease. J Radiol Case Reports. 2017;11:16-23.

6. Fitz RH. Persistent omphalo-mesenteric remains: their importance in the causation of intestinal duplication, cyst-formation, and obstruction. Am J Med Sci. 1884;88:30-57.

7. Doepker MP, Ahmad SA. Gastric duplication cyst: a rare entity. J Surg Case Reports. 2016;5:rjw073.

8. Tanaka M, Akahoshi K, Chijiiwa Y, Sasaki I, Nawata H. Diagnostic value of endoscopic ultrasonography in an unusual case of gastric cyst. Am J Gastroenterol. 1995;90:662-3.

9. Takahara T, Torigoe T, Haga H, Yoshida H, Takeshima S, Sano S, et al. Gastric duplication cyst: evaluation by endoscopic ultrasonography and magnetic resonance imaging. J Gastroenterol. 1996;31:420-4.

10. Scatizzi M, Calistri M, Feroci F, Girardi LR, Moraldi L, Rubio CA, et al. Gastric duplication cyst in an adult: case report. In Vivo. 2005;19:975-8.

11. Zhu Y, Lihong LV, Pan W, Ren P, Han T. Gastric duplication complicated by malignant transformation in adults: report of three cases. J Gastrointest Dig Syst. 2015;5:2.

12. Maeda H, Okabayashi T, Nishimori I, Kobayashi M, Morimoto K, Miyaji E et al. Diagnostic challenge to distinguish gastric duplication cyst from pancreatic cystic lesions in adult. Intern Med. 2007;46:1101-4. 